EPJ Web of Conferences 53, 09004 (2013)

DOI: $10.1051 /$ epjconf/20135309004

(C) Owned by the authors, published by EDP Sciences, 2013

\title{
Calibration of the JEM-EUSO detector
}

\author{
N. Sakaki ${ }^{1, a}$, J. Adams ${ }^{2}$, M. Christl ${ }^{3}$, P. Gorodetzky ${ }^{4}$ and L. Wiencke ${ }^{5}$ for the \\ JEM-EUSO collaboration ${ }^{\mathrm{b}}$
}

${ }^{1}$ Karlsruhe Institute of Technology (KIT), 76131 Karlsruhe, Germany

${ }^{2}$ University of Alabama in Huntsville, Huntsville, AL 35812, USA

${ }^{3}$ NASA - Marshall Space Flight Center, Huntsville, AL 35812, USA

${ }^{4}$ APC, Université Paris Diderot, 75013 Paris, France

${ }^{5}$ Department of Physics, Colorado School of Mines, Golden, CO 80401, USA

\begin{abstract}
In order to unveil the mystery of ultra high energy cosmic rays (UHECRs), JEM-EUSO (Extreme Universe Space Observatory on-board Japan Experiment Module) will observe extensive air showers induced by UHECRs from the International Space Station orbit with a huge acceptance. Calibration of the JEM-EUSO instrument, which consists of Fresnel optics and a focal surface detector with 5000 photomultipliers, is very important to discuss the origin of UHECRs precisely with the observed results. In this paper, the calibration before launch and on-orbit is described. The calibration before flight will be performed as precisely as possible with integrating spheres. In the orbit, the relative change of the performance will be checked regularly with on-board and on-ground light sources. The absolute calibration of photon detection efficiency may be performed with the moon, which is a stable light source in the nature.
\end{abstract}

\section{INTRODUCTION}

JEM-EUSO is a mission to observe ultra high energy cosmic rays (UHECRs) above $10^{20} \mathrm{eV}[1-4]$. The JEM-EUSO telescope will be attached to the International Space Station (ISS) to detect fluorescence photons in 300-430 $\mathrm{nm}$ range from extensive air showers (EASs) induced by UHECRs. It is very important to understand the instrument and the atmosphere to start particle astronomy as a pioneer. The telescope consists of the optics with three Fresnel lenses, one of which is diffractive, a focal surface with 5000 multi anode photomultiplier tubes (MAPMTs) and photon counting electronics [6]. Since the photon detection efficiency is related to the energy estimation directly, it is especially important to measure it accurately. And it is also important for the arrival direction determination, which is crucial for the astronomy. The number of observed photo-electrons $(\Delta S)$ from a luminous phenomena (with emitting number of photons of $\Delta Q$ ) at distance $r$ is expressed as:

$$
\Delta S=\frac{\epsilon \kappa \eta T_{l} T_{f} T_{e} T_{\alpha} A}{4 \pi r^{2}} \Delta Q
$$

where,

$\begin{array}{lllll}\epsilon & \text { quantum efficiency of the detector } & T_{f} & \text { transmission of the optical filter } \\ \eta & \text { collection efficiency of the detector } & T_{e} & \text { trigger efficiency of the electronics } \\ \kappa & \text { the probability to be contained in a pixel } & T_{\alpha} & \text { atmospheric transmission } \\ T_{l} & \text { throughput of the Fresnel lens system } & A & \text { aperture of the telescope. }\end{array}$

\footnotetext{
a e-mail: naoto.sakaki@kit.edu

bFor the full authorlist see Appendix "Collaborations" in this volume.
}

This is an Open Access article distributed under the terms of the Creative Commons Attribution License 2.0, which permits unrestricted use, distribution, and reproduction in any medium, provided the original work is properly cited. 


\section{EPJ Web of Conferences}

The terms related to the instrument among above are $\epsilon, \eta, \kappa, T_{f}, T_{e}$ and $T_{l}$. Here, let's consider the calibration of the instrument with the following four categories.

- Pre-flight calibration

- On-board calibration

- In-flight calibration with ground light sources

- Atmospheric monitor.

For monitoring the atmospheric condition in the field of view, especially around an EAS, a dedicated Atmospheric Monitoring System (AMS) is planned [5]. An ultra violet (UV) laser and an infrared camera are under preparation to measure the cloud coverage in the field of view and the cloud heights. Slow data of the JEM-EUSO instrument also gives information on the underlying cloud presence, in addition to the background intensity. The other three calibrations are included in the "calibration system" and are being prepared by the collaboration of Japan, Germany, France, United States, Italy and Mexico.

The calibration scheme is as follows.

1. Before the launch, the instrument will be characterized with accuracy better than 5\%. The characteristics will be stored into a data base.

2. Just after the launch, the end-to-end performance will be measured with a Xe flasher lamp on a NASA aircraft flying under the ISS.

3. The degradation of the instrument with time will be monitored with the following for methods.

- Degradation of the focal surface detector and the lenses will be measured with on-board light sources. Since the on-board resource is limited, the measurement of the efficiency will be limited to the relative change in principle, not the absolute scale. However, all gain changes will be measured absolutely.

- Absolute efficiency will be measured with Xe flasher on aircraft about once a month. The moon will be used as an absolute light source and the calibration accuracy will be $20 \%$.

- The reconstruction error on the arrival direction will be evaluated with on-ground lasers. The trigger efficiency for laser tracks will also be checked.

- Local atmospheric conditions at several places will be obtained with on-ground flashers to understand the atmosphere better together with on-board AMS devices.

The energy of $10^{20} \mathrm{eV}$ per particle has not been artificially achieved so far, so that it is difficult to determine the absolute energy scale directly by any calibration. It is the same for the other UHECR observatories on ground. However, if the energy spectrum for each source will be obtained with sufficient statistics, we may be able to see the dependence of the suppression energy (GZK effect), at which UHECRs interact with cosmic microwave background photons and lose an amount of energy that depends on the source distance. The position of the suppression energy observed by JEM-EUSO might lead to the absolute energy calibration of UHECR experiment.

\section{PRE-FLIGHT CALIBRATION}

\subsection{Overview}

The JEM-EUSO optics consists of two Fresnel lenses, a diffractive lens and a focal surface [6]. The focal surface is covered with about 5,000 MAPMTs (each $1^{\prime \prime}$ square) developed for JEM-EUSO. The focal surface detectors will be grouped to a module, to make it easy for manufacturing and the data handling. A flat module of four PMTs is called "Elementary Cell" (EC) and a curved photo-detector module (PDM) consists of 9 ECs. Each PDM has a capability to detect EASs induced by cosmic rays by itself. The stand-alone performance and functions of the optics, the electronics, etc. will be checked in 
UHECR 2012

(a)

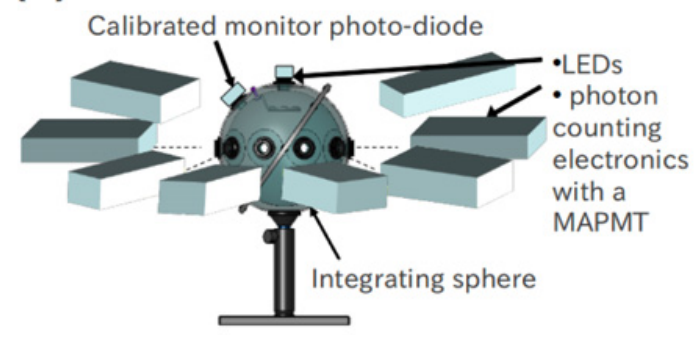

(b)

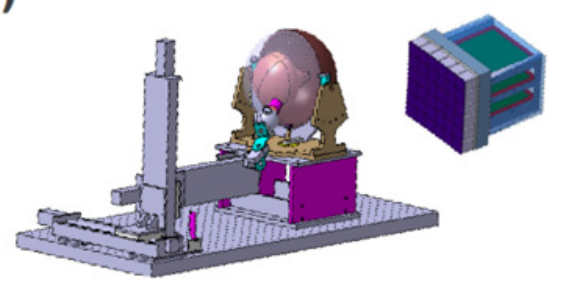

Figure 1. Apparatus for the pre-flight calibration. (a) Uniform light source made with an integrating sphere $\left(\cos ^{4} \theta\right.$ law) that illuminates ten MAPMTs. Single photo-electron spectrum will be taken with photo-electron counting and MAPMTs will be sorted according to the gain and the efficiency. (b) A light source made with an integrating sphere is mounted on a stage to measure the PDM efficiency at various positions and with various incident angles on the PMT surface.

each dedicated subsystem in principle. The calibration subsystem is in charge to measure the efficiency of the focal surface, especially efficiency of the MAPMTs.

\subsection{Calibration of MAPMTs}

The gain of a MAPMT will be measured at various voltages to determine the appropriate voltage for the input to the front-end electronics (a gain of $1.0 \times 10^{6}$ is required by the ASIC). An integrating sphere of $20 \mathrm{~cm}$ in diameter with 10 ports will be used to illuminate 10 MAPMTs at once. As shown in Figure 1(a), LED light will be diffused with the integrating sphere to illuminate the whole photocathodes of MAPMTs. A calibrated photo-diode attached on another port of the sphere will monitor the variation of the light intensity during the measurement. The size of the sphere ports and the distance between the sphere and the MAPMT will be optimized to make a uniform illumination over the whole photo-cathode. Three kinds of wavelength will be used in the range of $300 \mathrm{~nm}$ and $430 \mathrm{~nm}$. Pulse height distribution will be taken with the photo-electron counting method by sweeping the threshold level of the electronics developed for night glow measurement by the EUSO Italy group [7] or by the LAL ASIC test cards. The amount of the light emitted from a port is determined by the geometrical configuration, i.e. the ratio of the areas of the photo-diode and of the port [8]. The ratio will be measured in advance with two calibrated photo-diodes with more intense light than single photo-electron level. Since the photo-diode is precisely calibrated by the manufacturer, the amount of light at the photo-cathode can be known precisely enough. All the PMTs will be sorted out by their gain and efficiency, and then every four PMTs with similar characteristics will be packed as an EC.

\subsection{Calibration of PDMs}

In order to measure the position and angular dependencies of the photon detection efficiency of PDMs, the PDM surface will be scanned with a UV beam at $1 \mathrm{~mm}$ intervals and various incident angles. At this stage not only the efficiency of the MAPMTs themselves but also the performance of the JEMEUSO electronics is measured at the same time. Cross-talk will be also evaluated in this measurement. The light of a UV LED at $375 \mathrm{~nm}$ is diffused by an integrating sphere and is collimated to a parallel beam of $1 \mathrm{~mm} \phi$ size. The light source with the integrating sphere is mounted on a XYZ $\theta \phi$ stage and the PDM surface will be scanned (Fig. 1(b)). The light will be monitored by a precisely calibrated photo-diode attached to another port of the sphere. The efficiency (=[quantum efficiency] $\times$ [collection efficiency] $\times$ [electronics efficiency]) of not only MAPMTs but the whole system can be obtained. Therefore, the efficiency can be obtained better than a few percent. The data will be taken with the 
(a) Detector calibration

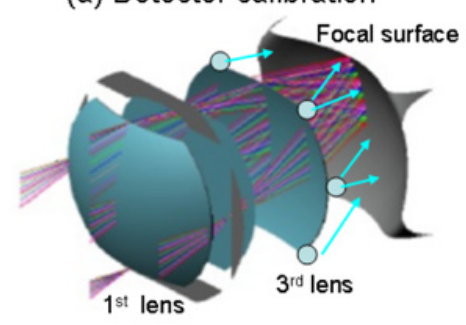

(b) Optics (+detector) calibration

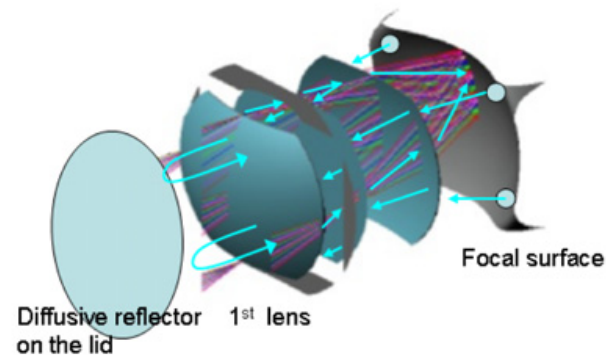

Figure 2. On-board calibration system. Diffused UV light sources made of integrating spheres will be set at the position shown in the panels (a) and (b), and the time variation of the efficiency of the optics and the detector will be monitored. (a) Several light sources will be set along the edge of the rear lens to illuminate the focal surface directly. The relative change of the detector efficiency will be taken. (b) The same light sources are placed along the edge of the focal surface to illuminate the rear lens. The light is reflected back at the diffuse surface on the lid and is detected by the focal surface detector. Here, convolution of the efficiency of the optics and that of the detector will be obtained.

photon-counting method in general, and several items like linearity check will be taken with more intense light. Another idea is to use the spot of an old analog oscilloscope.

Next, to check the trigger efficiency of PDM, fluorescence image from a cosmic ray air shower will be emulated with an array of UV LEDs. UV beam reflected by a rotating mirror is another candidate to emulate moving EAS tracks. The rotating speed will be adjusted to reproduce the light spot speed of EAS on the focal surface.

\section{IN-FLIGHT CALIBRATION}

\subsection{Overview}

Just after the JEM-EUSO launch, an end-to-end calibration on orbit will be performed by a Xe flasher lamp on an aircraft that underflys JEM-EUSO at $\sim 6 \mathrm{~km}$, possibly more than once, in the first month. The focusing quality and the total efficiency will be checked during this period. The molecular scattering is expected to be dominant above that altitude, so that the amount of light at the entrance pupil can be calculated with small error. On a regular basis, only the relative changes will be monitored in flight in principle. Several light sources will be placed in the JEM-EUSO instrument to monitor the efficiency and the detector gain. That measurement will be performed in the daylight segments of the ISS orbit with the lid closed. The global light system (GLS) is a worldwide network of Xe flasher stations and ground laser stations which are remotely controlled [9]. A dozen of units will be deployed all over the world between $\pm 51.6^{\circ}$ latitude. On average one of stations will be viewed by JEM-EUSO every day providing a data base that will be sensitive to seasonal variations at the locations. The reflection of the moonlight by the Earth will also be utilized to make the calibration on the absolute photon detection efficiency more reliable.

\subsection{On-board calibration}

The light source consists of a small integrating sphere equipped with UV LED in 300-430 nm and a calibrated photo-diode to monitor the variation of the light intensity. Several identical light sources will be located behind the rear lens and illuminate the whole focal surface (Fig. 2 (a)). The intensity will be set at single photo-electron level and the photon detection efficiency of the system will be obtained in 
relative way, while the gain of MAPMT will be measured absolutely. If large change of gain is found, the threshold level for the counting will be adjusted.

Other several light sources will be set along the edge of the focal surface to illuminate the rear lens (Fig. 2 (b)). The light passes through the lenses and is reflected back at the diffuse surface on the lid. A certain amount of the emitted light will be detected by the focal surface detector. Sand-blasted aluminum is the most probable candidate for the diffuse surface from the view of resistance to space environment. The time variation of the performance of the optics and the detector will be obtained at the same time in this measurement. Therefore, after subtracting the degradation of the detector itself, the change in the optics throughput will be obtained.

\subsection{From-ground calibration: Global Light System (GLS)}

\subsubsection{Overview}

As described in Sect. 3.1, 12 units of ground based light system will be placed around the world. All of them have Xe flashers and 6 of them have also steerable lasers described in the following sections. The locations will be chosen for their low background and the altitude to avoid the effect of Mie scattering by dust in the boundary layer as much as possible. Atmospheric condition will be monitored with flashers and reconstruction quality of the EAS events will be evaluated with lasers in various atmospheric conditions. Balloon flights are planned to demonstrate a prototype of the JEM-EUSO telescope [10]. The balloon experiment targets the proof of the end-to-end JEM-EUSO techniques, measurement of background and the acquisition of real cosmic ray data above $10^{18} \mathrm{eV}$. A prototype of the airborne GLS will be deployed to proof the GLS concept.

\subsubsection{GLS flasher}

The flasher will be used to cross-check the JEM-EUSO photon detection efficiency, atmospheric transmittance and focusing quality of the JEM-EUSO optics. The GLS Flasher is equipped with a Xe flash lamp (Hamamatsu L6604) with filters of four wavebands: a wide bandpass filter in the range of 300-430 nm (Schott BG3) and three narrow bandpass filters at 337, 357 and $391 \mathrm{~nm}$ which are the main fluorescence lines of nitrogen. The flashers are housed in a weather-tight enclosure that includes the flasher, power supplies, battery and data acquisition electronics. They will be operated by solar batteries and be connected to the Internet for the remote control. The lamp flashes at $10 \mathrm{~Hz}$ and is expected to $<3 \%$ degradation during the mission period. The flash-to-flash variation is less than $3 \%$ and monitored with a calibrated photo-diode. The light will be diffused and the intensity varies slowly up to the field $>60^{\circ}$. The duration of over-flight is 56 seconds on average. Since the GLS flashers can be seen as a point light source by JEM-EUSO, the size of the image spreads with the point spread function of the optics. The size of the image will be monitored during the mission period. If the spot becomes remarkably large, commands can be sent to adjust the distance between the lens and the focal surface to improve the focus.

\subsubsection{GLS laser}

Six GLS units [9] will have steered $355 \mathrm{~nm}$ NdYAG laser systems to emulate the optical signatuers of EASs. There is an approximate effective optical equivalence between a $5 \mathrm{~mJ} 355 \mathrm{~nm}$ laser track and a $10^{20} \mathrm{eV}$ air shower track [11] Ground based laser systems have been used successfully at the Fly's Eye [12], HiRes [13, 14], the Telescope Array [15], and the Pierre Auger Observatory (Auger) [16, 17]. The GLS laser units will use many of the components used in the Pierre Auger systems [18].

By adjusting the GLS laser energy and direction, the triggering threshold of JEM-EUSO will be tested. GLS laser will also be aimed at a selected set of UHECR potential sources over the full sky. 
Examples will include the galactic center, Cen A, Virgo, and other objects of astrophysical interest. A sky map of reconstructed laser track directions will be accumulated over the mission. The clusters of points will provide a simple but comprehensive validation of the absolute EAS pointing accuracy reconstruction by JEM-EUSO. Included in this test is transfer of time stamps from a precise clock on ISS through the JEM-EUSO hardware and data analysis chains. During clear periods when UV light propagation between the GLS site and the ISS can be described to the few percent level using molecular scattering alone, it will be possible to test intrinsic luminosity with both the direct Xe flasher light (point source) and the scattered UV laser light (track source). These periods can be identified by comparing the ratio of flasher measurements at different viewing angles by JEM-EUSO to the ratio predicted by molecular scattering together with measurements of the JEM-EUSO IR camera and on-board laser.

\subsubsection{Airborne GLS}

An airborne Xe flasher unit is to be installed on an upward directed portal of a P3B research aircraft stationed at Wallops Flight Facility/NASA. There is a side port to be fitted for the transmittance of a horizontal UV laser. The flasher unit has same functions as the on-ground flashers. As mentioned in Sect. 3.1, the airborne flasher will be used intensively for several times for the measurement of the endto-end efficiency after the launch. It flies under the orbit of ISS at the altitude of $1-6 \mathrm{~km}$ above both land and sea every month during the mission period to measure the atmospheric condition in various seasons and the end-to-end efficiency of the JEM-EUSO instrument.

\section{SUMMARY}

Calibration of the instrument plays a key role in opening a new era of "Particle Astronomy" by JEMEUSO. In order to achieve better than a few \% accuracy in total, combination of several methods for pre-flight and in-flight calibrations (on-board and with ground light sources) are proposed and the preparation is in progress.

This project was partially supported by JSPS and CNRS under the Japan-France Research Cooperative Program.

\section{References}

[1] M. Casolino et al., [JEM-EUSO collab.], Astrophys. Space Sci. Trans. 7 (2011) 477

[2] A. Santangelo et al., [JEM-EUSO collab.], This symposium proceedings

[3] M. Bertaina et al., [JEM-EUSO collab.], This symposium proceedings

[4] G. Medina Tanco et al., [JEM-EUSO collab.], This symposium proceedings

[5] M.D. Rodriguez Frias et al., [JEM-EUSO collab.], This symposium proceedings

[6] F. Kajino et al., [JEM-EUSO collab.], Nucl. Instr. Meth. A 623 (2010) 422

[7] M.C. Maccarone et al., Nucl. Inst. Meth. A 659 (2011) 569

[8] G. Lefeuvre, Ph.D. thesis "Precise measurement of the Absolute Yield of the Fluorescence of Nitrogen in the Air. Effects on the Detection of the Cosmic rays of Ultra-high Energy" (University Paris 7), (2006)

[9] J. Adams et al., (2012) [arXiv:1203.3451v2 [astro-ph.IM]]

[10] P. von Ballmoos et al., [JEM-EUSO collab.], This symposium proceedings

[11] L. Wiencke [for the Pierre Auger Collab.], J. Phys. Conf. Ser. 160 (2009) 012037. [arXiv:0807.2884 [astro-ph]]

[12] D. J. Bird et al., Astrophys. J. 441 (1995) 144

[13] L. Wiencke et al., in Proceedings of SPIE 3818 (1999) 46 


\section{UHECR 2012}

[14] C. Cannon, L. Pedersen, R. Riehle, J. Thomas and L. Wiencke [for the HiRes Collab.], Proc. 28th ICRC (2003) 447

[15] H. Kawai et al., Nucl. Phys. Proc. Suppl. 175-176 (2008) 253

[16] J. Abraham et al., [Pierre Auger Collab.], Astropart. Phys. 33 (2010) 108. [arXiv:1002.0366 [astro-ph.IM]]

[17] P. Abreu et al., [Pierre Auger Collab.], Proc. 32nd ICRC, (2011) [arXiv:1107.4806v1 [astro-ph.IM]]

[18] B. Fick et al., JINST 1 (2006) P11003. [arXiv:astro-ph/0507334v1] 